\title{
Cribriform growth is highly predictive for postoperative metastasis and disease-specific death in Gleason score 7 prostate cancer
}

\author{
Charlotte F Kweldam ${ }^{1}$, Mark F Wildhagen ${ }^{2,3}$, Ewout W Steyerberg ${ }^{4}$, Chris H Bangma ${ }^{3}$, \\ Theodorus $\mathrm{H}$ van der Kwast ${ }^{5}$ and Geert JLH van Leenders ${ }^{1}$ \\ ${ }^{1}$ Department of Pathology, Erasmus Medical Center, Rotterdam, The Netherlands; ${ }^{2}$ Research Office Sophia, \\ Erasmus Medical Center, Rotterdam, The Netherlands; ${ }^{3}$ Department of Urology, Erasmus Medical Center, \\ Rotterdam, The Netherlands; ${ }^{4}$ Center for Medical Decision Making, Erasmus Medical Center, Rotterdam, \\ The Netherlands and ${ }^{5}$ Department of Pathology and Laboratory Medicine, University Health Network, \\ Toronto, Canada
}

\begin{abstract}
Patients with Gleason score 7 prostate cancer on radical prostatectomy demonstrate a wide range in clinical outcome. Gleason grade 4 prostate cancer encompasses a heterogeneous group of tumor growth patterns including fused, ill-defined, cribriform, and glomeruloid glandular structures. Our objective was to determine the prognostic value of different Gleason grade 4 growth patterns. We performed a nested case-control study among 535 patients with Gleason score 7 prostate cancer at radical prostatectomy, treated between March 1985 and July 2013 at a university hospital in the Netherlands. We analyzed 52 cases (with metastasis, diseasespecific mortality or both) and 109 controls, matched for age, PSA level, and pT stage. Presence of the following Gleason grade 4 patterns was recorded: fused, ill-defined, cribriform, and glomeruloid. Intraductal carcinoma of the prostate and tertiary Gleason grade 5 were additionally assessed. Outcomes were metastasis-free survival and disease-specific survival. We used Cox proportional hazards regression to determine the predictive value of Gleason grade 4 patterns for survival time. The overall prevalence of Gleason grade 4 patterns was as follows: fused $75 \%(n=121)$, ill-defined $64 \%(n=102)$, cribriform $48 \%(n=83)$, and glomeruloid $25 \%(n=40)$. Cribriform pattern was the only pattern with an unequal distribution between cases and controls. Forty-two out of 52 cases $(81 \%)$ had cribriform growth pattern versus $41 / 109$ controls $(38 \%)$. In multivariate analysis, presence of cribriform growth was an adverse independent predictor for distant metastasis-free survival $(\mathrm{HR} 8.0,95 \% \mathrm{Cl}$ 3.0-21; $P<0.001$ ) and disease-specific survival (HR 5.4, 95\% Cl 2.0-15, $P=0.001$ ). In conclusion, cribriform growth in Gleason grade 4 is a strong prognostic marker for distant metastasis and disease-specific death in patients with Gleason score 7 prostate cancer at radical prostatectomy.

Modern Pathology (2015) 28, 457-464; doi:10.1038/modpathol.2014.116; published online 5 September 2014
\end{abstract}

The widely used Gleason grading system for prostate cancer discerns five different grades based on the architectural tumor growth pattern. ${ }^{1}$ The Gleason score is determined by adding the two most common Gleason grades in radical prostatectomies; in needle-biopsies the most common and highest Gleason grades are added. The Gleason grading system is an important predictor of disease progression, and one of the most important

Correspondence: Dr CF Kweldam, MD, Department of Pathology, Erasmus Medical Center, Postbus 2040, 3000CA Rotterdam, The Netherlands.

E-mail: c.kweldam@erasmusmc.nl

Received 17 June 2014; revised 11 July 2014; accepted 12 July 2014; published online 5 September 2014 parameters for clinical decision making. In 2005, large cribriform and ill-defined glands, classically described as Gleason grade 3, were redefined as Gleason grade $4 .^{2}$ Later, small cribriform and glomeruloid glands have been reconsidered Gleason grade 4 as well. ${ }^{3-5}$ This grade migration has led to a decline in reporting of Gleason score 6 on radical prostatectomy, joined by a relative increase of Gleason score 7 prostate cancer. ${ }^{6}$ Whereas patients with modified Gleason score 6 on radical prostatectomy represent a group with excellent outcome, patients with Gleason score 7 demonstrate a wide range in clinical outcome. ${ }^{7-9}$ Risk stratification within the Gleason score 7 patient population remains a challenge, and additional prognostic factors are needed. The objective of this study was 
to determine the predictive value of distinctive Gleason grade 4 growth patterns for metastasis and disease-specific death in men with Gleason score 7 prostate cancer on radical prostatectomy.

\section{Materials and methods}

\section{Study Design}

We identified 535 hormone-naive patients with Gleason score 7 prostate cancer on radical prostatectomy, treated between March 1985 and July 2013 at the Erasmus MC, Rotterdam, the Netherlands. In our cohort, 56 patients had documented metastasis or disease-specific death during follow-up ('cases'). The control group consisted of 112 Gleason score 7 patients without documented metastasis or diseasespecific death. We matched the control group for the following three parameters: age at time of surgery, serum prostate-specific antigen (PSA) level at time of diagnosis $(\mathrm{ng} / \mathrm{ml})$, and pT stage. ${ }^{10}$ We randomly selected controls in the pT2 and pT3a group with follow-up $\geq 120$ months. Limits for age were $\geq 47$ and $\leq 74$ years. Limits for PSA level were $\geq 0$ and $\leq 100 \mathrm{ng} / \mathrm{ml}$. In 7 patients, histopathologic slides and blocks could not be retrieved from the archive (4 cases and 3 controls), leaving 52 cases and 109 controls for analysis with all slides and clinicopathologic information available.

\section{Pathologic Evaluation}

After operation, all radical prostatectomy specimens were routinely examined at the Department of Pathology of our institute. At pathologic evaluation, Gleason score, pT stage, and surgical margin status were recorded for each patient. From 1985 to 2005, the classic Gleason grading system was applied; the modified Gleason grading was used after 2005. ${ }^{1,2,11,12}$ The 2009 TNM classification was used to assess pT stage. ${ }^{10}$ A positive surgical margin was defined as extension of the tumor into the inked surface of the specimen.

The investigator (CFK) and a board-certified pathologist with expertise in urogenital pathology (GJLHvL) reviewed all slides and routinely determined the modified Gleason score. ${ }^{2,5}$ Both reviewers were blinded to the patients' outcome. The presence of Gleason grade 4 growth patterns was specifically recorded in each specimen. In addition, we assessed the presence of tertiary Gleason grade 5 and intraductal carcinoma of the prostate in each specimen as both have been associated with adverse clinical outcome. ${ }^{13-17}$ The following Gleason grade 4 growth patterns, as defined by the ISUP-modified Gleason grading scheme, ${ }^{2}$ were scored: (1) fused glands included fused well- and poorly formed glands (Figure 1a). (2) Ill-defined glands comprised glands with poorly formed or absent glandular lumina (Figure 1b). Only a cluster of such glands was acceptable, to exclude the possibility of tangentially sectioned Gleason pattern 3 glands. (3) Cribriform was characterized by a solid proliferation with multiple punched-out lumina, without intervening stroma (Figure 1c). (4) Glomeruloid glands were defined as the presence of dilated glands containing a cribriform proliferation that is attached to only one edge of the gland, resulting in the structure resembling a glomerulus (Figure 1d). Tertiary Gleason grade 5 was defined as presence of (1) solid sheets, cords, or single cells with no glandular differentiation or (2) comedocarcinoma with central necrosis surrounded by papillary, cribriform, or solid masses in $<5 \%$ of the radical prostatectomy specimen. Intraductal carcinoma was defined as a well-circumscribed lesion surrounded by an intact basal cell layer distended by overtly malignantappearing epithelial populations (Figure 1e). ${ }^{18}$ Clear distention of prostate glands and presence of necrosis were used as cutoffs to distinguish intraductal carcinoma from high-grade prostatic epithelial neoplasia. To distinguish small foci of cribriform pattern from fused glands we applied two criteria. Contact of the majority of tumor cells with adjacent stroma (1) and more linear orientation of lumina instead of rounded lumina (2) were both in favor for fused glands. In addition, we did not use a size threshold for cribriform fields. When cribriform and intraductal carcinoma could not be distinguished morphologically, immunohistochemistry for basal cells (34BE12) was performed $(n=6)$; presence of basal cells was considered supportive of intraductal carcinoma (Figure 1f). Consensus was reached in all cases during a joint session.

\section{Follow-Up}

After surgery, patients were monitored annually at our out-patient clinic. Biochemical recurrence was defined as a PSA level of $\geq 0.2 \mathrm{ng} / \mathrm{ml}$, assessed at two consecutive time points $>3$ months apart after radical prostatectomy. Metastasis was defined as presence of prostate cancer in a lymph node or at a distant site, with radiologic or pathologic confirmation. As all lymph node metastases in this cohort were diagnosed at time of operation (follow-up 0 months), they were not included as an end point but as a covariate in multivariate analysis of distant metastasis and disease-specific death. Distant metastases in this study were all hematogenous. Outcome variables were biochemical recurrence-free survival defined as time after radical prostatectomy to biochemical recurrence; metastasis-free survival defined as time after radical prostatectomy to distant metastasis; disease-specific survival defined as time after radical prostatectomy to death attributed to prostate cancer; overall survival defined as time after radical prostatectomy to all-cause death. Death and disease-specific death were administrated by medical record review and death certificates. All 

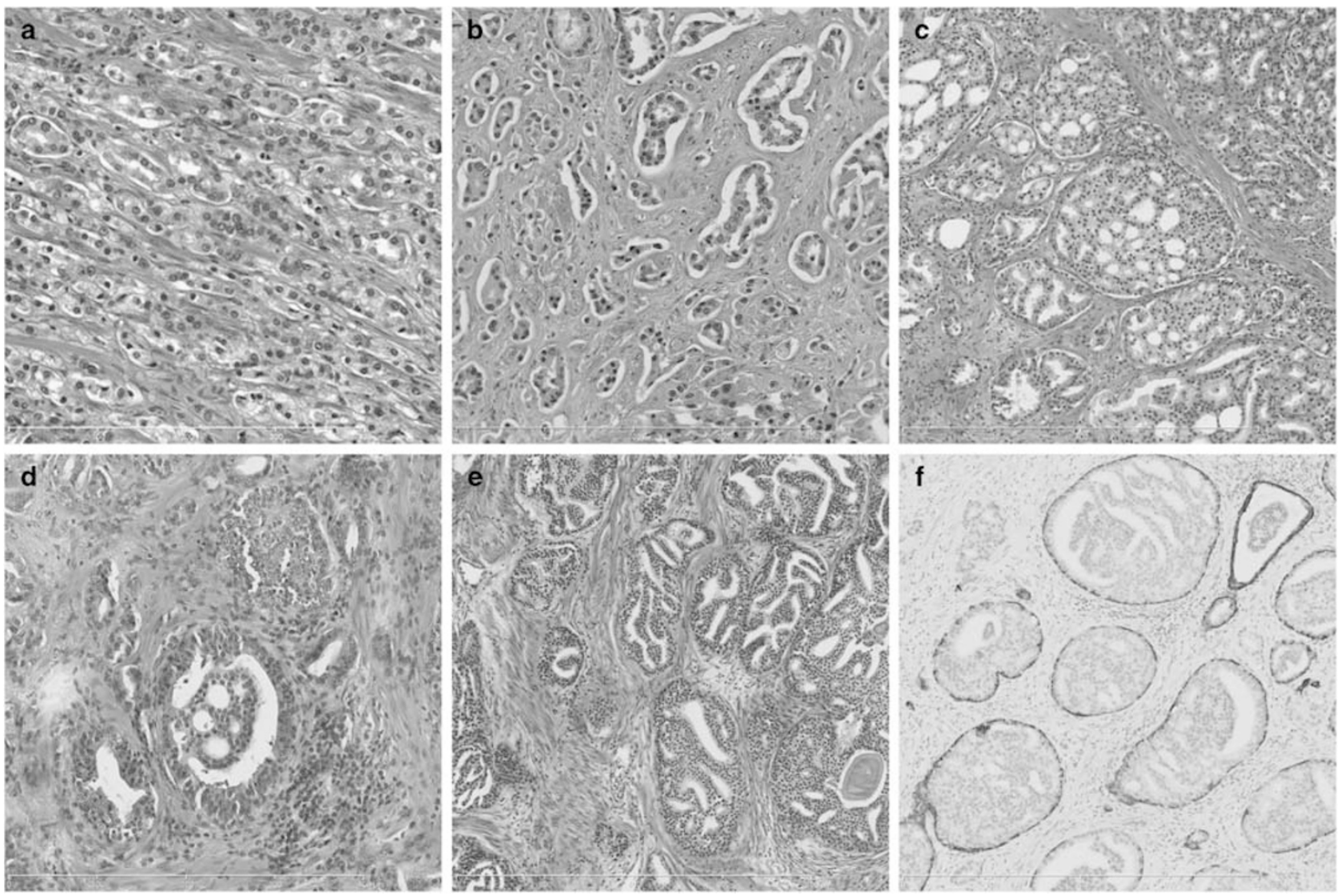

Figure 1 Gleason grade 4 patterns and intraductal carcinoma. (a) Fused glands; (b) ill-defined glands; (c) cribriform glands; (d) glomeruloid gland; (e) intraductal carcinoma; and (f) 34BE12 immunohistochemistry, demonstrating the presence of basal cells supportive for intraductal carcinoma.

relevant clinical, pathologic, and follow-up data were recorded and regularly updated in a prospective study database (MW).

\section{Statistics}

Continuous clinicopathologic parameters were analyzed using the Mann-Whitney $U$-test, and categorical parameters using the Pearson's $\chi^{2}$ test. Correlation coefficients were calculated by the Spearman's rank correlation test. Survival probabilities were estimated by the Kaplan-Meier method. Unadjusted two-group comparisons for survival time were made with log-rank testing. We used Cox proportional hazards regression to determine the predictive value of Gleason grade 4 patterns for survival time. Age, PSA level, Gleason score, pT stage, surgical margin status, lymph node status, Gleason grade 4 patterns, intraductal carcinoma, and tertiary Gleason grade 5 were all included in the multivariable analysis as potential confounders. Dummy variables were created to convert pT stage into series of binary groups. All statistics were performed using SPSS 22 (SPSS, Chicago, IL, USA). A two-sided $P$-value $<0.05$ was considered significant.

\section{Results}

\section{Patient Characteristics}

The clinicopathologic characteristics are listed in Table 1. The median follow-up in controls was 160 months (IQR 120-190), which was significantly longer than the follow-up of the cases (100 months; IQR 78-150; $P=0.001)$. As expected from the matching, cases and controls had a similar distribution for age, PSA level and pT stage (Table 1). Gleason score $4+3=7$ was more frequent in cases than controls ( $48 \%$ vs $\left.19 \%, \chi^{2} P=0.001\right)$. Furthermore, cribriform pattern and intraductal carcinoma were both more often present in cases than controls: $81 \%$ vs $38 \% \quad\left(\chi^{2} \quad P<0.001\right)$ and $58 \% \quad$ vs $33 \%$ $\left(\chi^{2} P=0.003\right)$, respectively. Cases and controls showed a similar distribution for fused, ill-defined, and glomeruloid Gleason grade 4 patterns, tertiary Gleason grade 5, number of Gleason grade 4 patterns, and surgical margin status.

Biochemical recurrence occurred in 44/52 (85\%) of the cases and in 45/109 (41\%) of the controls. In cases, the median time to biochemical recurrence was 19 months (IQR 11-37); in controls this was 61 months (IQR 28-110) (log rank $P<0.001$ ). A total of $45(87 \%)$ cases had metastatic disease, of which 11 
Table 1 Clinicopathologic characteristics of the entire study population $(N=535)$, cases $(n=52)$ and controls $(n=109)$

\begin{tabular}{|c|c|c|c|c|}
\hline Clinicopathologic parameter & Entire cohort $(\mathrm{N}=535)$ & Cases $(\mathrm{n}=52)$ & Controls $(\mathrm{n}=109)$ & P-value ${ }^{\mathrm{a}}$ \\
\hline Age at time of surgery (years) & $64(65 ; 60-68)$ & $63(63 ; 60-68)$ & 63 (59-68) & $0.76^{\mathrm{b}}$ \\
\hline PSA level $(\mathrm{ng} / \mathrm{ml})$ & $9.8(6.4 ; 4.2-10)$ & $12(7.8 ; 5.3-13)$ & $12(7.4 ; 5.4-16)$ & $0.60^{\mathrm{b}}$ \\
\hline Follow-up after radical prostatectomy (months) & $94(91 ; 37-150)$ & $110(100 ; 78-150)$ & $140(160 ; 120-190)$ & $0.001^{\mathrm{b}}$ \\
\hline \multicolumn{5}{|l|}{ Gleason score } \\
\hline $3+4$ & $436(81)$ & $27(52)$ & $88(81)$ & \multirow[t]{2}{*}{$0.001^{\circ}$} \\
\hline $4+3$ & $99(19)$ & $25(48)$ & $21(19)$ & \\
\hline \multicolumn{5}{|l|}{ pT stage (2009) } \\
\hline $\mathrm{T} 2$ & $270(50)$ & $10(19)$ & $22(20)$ & \multirow[t]{3}{*}{$0.48^{\mathrm{C}}$} \\
\hline Т3а & $218(41)$ & $25(48)$ & $61(56)$ & \\
\hline T3b & $47(8.8)$ & $17(33)$ & $26(24)$ & \\
\hline Cribriform growth pattern & & $42(81)$ & $41(38)$ & $<0.001^{\mathrm{C}}$ \\
\hline Fused & & $38(73)$ & $83(76)$ & $0.67^{\mathrm{C}}$ \\
\hline Ill-defined & & $30(58)$ & $72(66)$ & $0.30^{\mathrm{C}}$ \\
\hline Glomeruloid & & $9(17)$ & $31(28)$ & $0.13^{\mathrm{C}}$ \\
\hline \multicolumn{5}{|l|}{ Number of Gleason grade 4 patterns } \\
\hline 1 & & $31(24)$ & $4(13)$ & \multirow[t]{4}{*}{$0.16^{\mathrm{b}}$} \\
\hline 2 & & $58(45)$ & $17(53)$ & \\
\hline 3 & & $33(26)$ & $9(28)$ & \\
\hline 4 & & $7(5.4)$ & $2(6.2)$ & \\
\hline Intraductal carcinoma & & $30(58)$ & $36(33)$ & $0.003^{\mathrm{C}}$ \\
\hline Tertiary Gleason grade 5 & & $5(9.6)$ & $8(7.3)$ & $0.62^{\mathrm{C}}$ \\
\hline
\end{tabular}

Values denote either mean (median; IQR) or $n(\%)$.

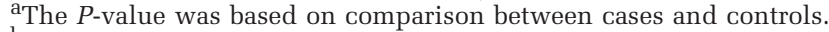

bMann-Whitney $U$-test.

CPearson's $\chi^{2}$ test.

were lymph node metastasis $(21 \%)$ all discovered at time of operation, and 37 distant metastasis (71\%). The median time to distant metastasis was 67 months (IQR 46-94). Thirty men (60\%) of the cases died from prostate cancer. The median time to disease-specific death was 110 months (IQR 91140). The overall mortality rate was $70 \%$ in cases and $14 \%$ in controls.

\section{Cribriform Growth Pattern Is Associated with Gleason Score $4+3=7$ and Intraductal Carcinoma}

The clinicopathologic characteristics of the study population stratified by either the presence or absence of cribriform pattern are listed in Table 2 . Presence of cribriform pattern was three times more frequent in patients with Gleason score $4+3=7$ ( $42 \%$ vs $14 \%$ in the absence of cribriform pattern, $\left.\chi^{2} P<0.001\right)$. Intraductal carcinoma was seen in $54 /$ 83 patients with cribriform pattern $(65 \%)$ and vice versa cribriform pattern in 54/66 (82\%) patients with intraductal carcinoma (Spearman's $\rho=0.51$, $P<0.001$ ), whereas intraductal carcinoma without cribriform pattern occurred in 12/78 patients (15\%). In addition, cribriform pattern was positively associated with presence of multiple Gleason grade 4 patterns (Spearman's $\rho=0.54, P<0.001$ ). Also, lymph node metastases were more prevalent in specimens with cribriform pattern $(11 \%$ vs $2.6 \%$ in the absence of cribriform pattern, $\chi^{2} P=0.037$ ). By contrast, ill-defined glands were more prevalent when cribriform pattern was absent $(76 \%$ vs $52 \%$; $\left.\chi^{2} P=0.002\right)$.

\section{Predictors for Biochemical Recurrence: Seminal Vesicle Invasion, Positive Surgical Margins and Cribriform Pattern}

When cribriform pattern was present, the median time to biochemical recurrence was 34 months (IQR 11-88), and 120 months (IQR 40-170) when cribriform pattern was absent (log rank $P<0.001$ ) (Figure 2a). Age, PSA level, Gleason score $4+3=7$, seminal vesicle invasion (pT3b), positive surgical margins, and intraductal carcinoma were significant predictors for biochemical recurrence-free survival in a univariate analysis (data not shown). In multivariable analysis the following three parameters were independent predictors for biochemical recurrence-free survival: seminal vesicle invasion (HR 2.6, 95\% CI 1.2-5.7, $P=0.014)$, positive surgical margins (HR 1.9, 95\% CI 1.2-3.0, $P=0.010$ ), and cribriform pattern (HR 2.0, 95\% CI 1.2-3.4, $P=0.006)$.

\section{Cribriform Pattern Is a Strong Predictor for Distant Metastasis and Disease-Specific Death}

The median time to distant metastasis in patients with cribriform pattern was 88 months (IQR 42-160) and 150 months in men without cribriform pattern (IQR 120-180, log rank $P<0.001$ ) (Figure 2b). Crude 
Table 2 Clinicopathologic characteristics of the study population $(n=161)$ stratified by cribriform growth pattern

\begin{tabular}{|c|c|c|c|}
\hline \multirow{2}{*}{ Clinicopathologic parameter } & \multicolumn{2}{|c|}{ Cribriform growth pattern } & \multirow{2}{*}{$\mathrm{P}$-value } \\
\hline & Present $(\mathrm{n}=83)$ & Absent $(\mathrm{n}=78)$ & \\
\hline Age at time of surgery (years) & $63(63 ; 59-68)$ & $63(64 ; 60-68)$ & $0.30^{\mathrm{a}}$ \\
\hline PSA level (ng/ml) & $14(8.1 ; 5.4-17)$ & $9.1(7.1 ; 5.2-12)$ & $0.15^{\mathrm{a}}$ \\
\hline Follow-up after radical prostatectomy (months) & $120(120 ; 73-170)$ & $140(150 ; 120-180)$ & $0.012^{\mathrm{a}}$ \\
\hline \multicolumn{4}{|l|}{ Gleason score } \\
\hline $3+4=7$ & $48(58)$ & $67(86)$ & \multirow[t]{2}{*}{$<0.001^{\mathrm{b}}$} \\
\hline $4+3=7$ & $35(42)$ & $11(14)$ & \\
\hline \multicolumn{4}{|l|}{ pT stage (2009) } \\
\hline $\mathrm{T} 2$ & $14(17)$ & $18(23)$ & \multirow[t]{3}{*}{$0.33^{\mathrm{b}}$} \\
\hline T3a & $43(52)$ & $43(55)$ & \\
\hline T3b & $26(31)$ & $17(22)$ & \\
\hline Positive surgical margin & $35(43)$ & $45(46)$ & $0.78^{\mathrm{b}}$ \\
\hline Fused & $66(80)$ & $55(71)$ & $0.19^{\mathrm{b}}$ \\
\hline Ill-defined & $43(52)$ & $59(76)$ & $0.002^{\mathrm{b}}$ \\
\hline Glomeruloid & $22(27)$ & $18(23)$ & $0.62^{\mathrm{b}}$ \\
\hline \multicolumn{4}{|l|}{ Number of Gleason grade 4 patterns } \\
\hline 1 & $6(7.2)$ & $29(37)$ & \multirow[t]{4}{*}{$<0.001^{\mathrm{a}}$} \\
\hline 2 & $32(39)$ & $43(55)$ & \\
\hline 3 & $36(43)$ & $6(7.7)$ & \\
\hline 4 & 9 (11) & $0(0.0)$ & \\
\hline Intraductal carcinoma & $54(65)$ & $12(15)$ & $<0.001^{\mathrm{a}}$ \\
\hline Tertiary Gleason grade 5 & $11(13)$ & $2(2.6)$ & $0.013^{b}$ \\
\hline
\end{tabular}

Values denote either mean (median; IQR) or $n(\%)$.

${ }^{\mathrm{a}}$ Mann-Whitney $U$-test.

bearson's $\chi^{2}$ test.

and adjusted hazard ratios for distant metastasis are listed in Table 3. Univariate predictors for distant metastasis were Gleason score $4+3=7$, cribriform pattern, intraductal carcinoma, and tertiary Gleason grade 5; in multivariable analysis cribriform pattern was the only independent predictor for distant metastasis (HR 8.0, 95\% CI 3.0-21, $P<0.001$ ), adjusted for age, PSA level, Gleason score, pT stage, surgical margin status, lymph node status, Gleason grade 4 patterns, intraductal carcinoma, and tertiary Gleason grade 5. In contrast, fused pattern was associated with a decreased adjusted hazard ratio for distant metastasis (HR 0.47, 95\% CI 0.22-1.0, $P=0.048)$. The median time to disease-specific death in men with cribriform pattern was 120 months (IQR 76-170) and 150 months (IQR 120180 ) in men without cribriform pattern (log rank $P<0.001$ ) (Figure 2c). Crude and adjusted hazard ratios for disease-specific survival are listed in Table 3. Univariate predictors for disease-specific survival were Gleason score $4+3=7$, cribriform pattern, and intraductal carcinoma (Table 3). Independent predictors for disease-specific survival were both Gleason score $4+3=7$ (HR 3.1, 95\% CI 1.4-7.1, $P=0.007$ ) and cribriform pattern (HR 5.4, 95\% CI 2.0-15, $P=0.001)$. Furthermore, the overall survival in patients with cribriform pattern was shorter than in patients without cribriform pattern (log rank $P=0.001$ ) (Figure 2d).
Cribriform Pattern in Gleason Score 3 $+4=7$ and Gleason Score $4+3=7$ Subpopulations

Primary Gleason grade 4 (Gleason score $4+3=7$ ) was unequally distributed between cases and controls (Table 1). Although Cox regression analysis compensated for this unequal distribution, we additionally studied the predictive value of cribriform pattern separately in Gleason score $3+4=7$ $(n=115)$ and Gleason score $4+3=7(n=46)$ subpopulations. In Gleason score $3+4=7$, cribriform pattern was an independent predictor for both distant metastasis-free survival (20 events; HR 6.0, 95\% CI 2.0-18, $P=0.001$, log rank $P<0.001)$ and disease-specific survival (13 events; HR 4.9, 95\% CI $1.3-18, P=0.017$, log rank $P=0.013$ ) in multivariable analysis. Owing to the limited number of events $(n=12)$ and number of covariates, no models could be fit for distant metastasis-free survival in Gleason score $4+3=7$ prostate cancer patients. Cribriform pattern was, however, an independent predictor for disease-specific survival (12 events) in Gleason score $4+3=7$ (HR 17, 95\% CI 2.2-130, $P=0.006$ ).

\section{Discussion}

The clinical outcome of Gleason score 7 prostate cancer after radical prostatectomy is highly variable. Pathologically, Gleason grade 4 prostate cancer 

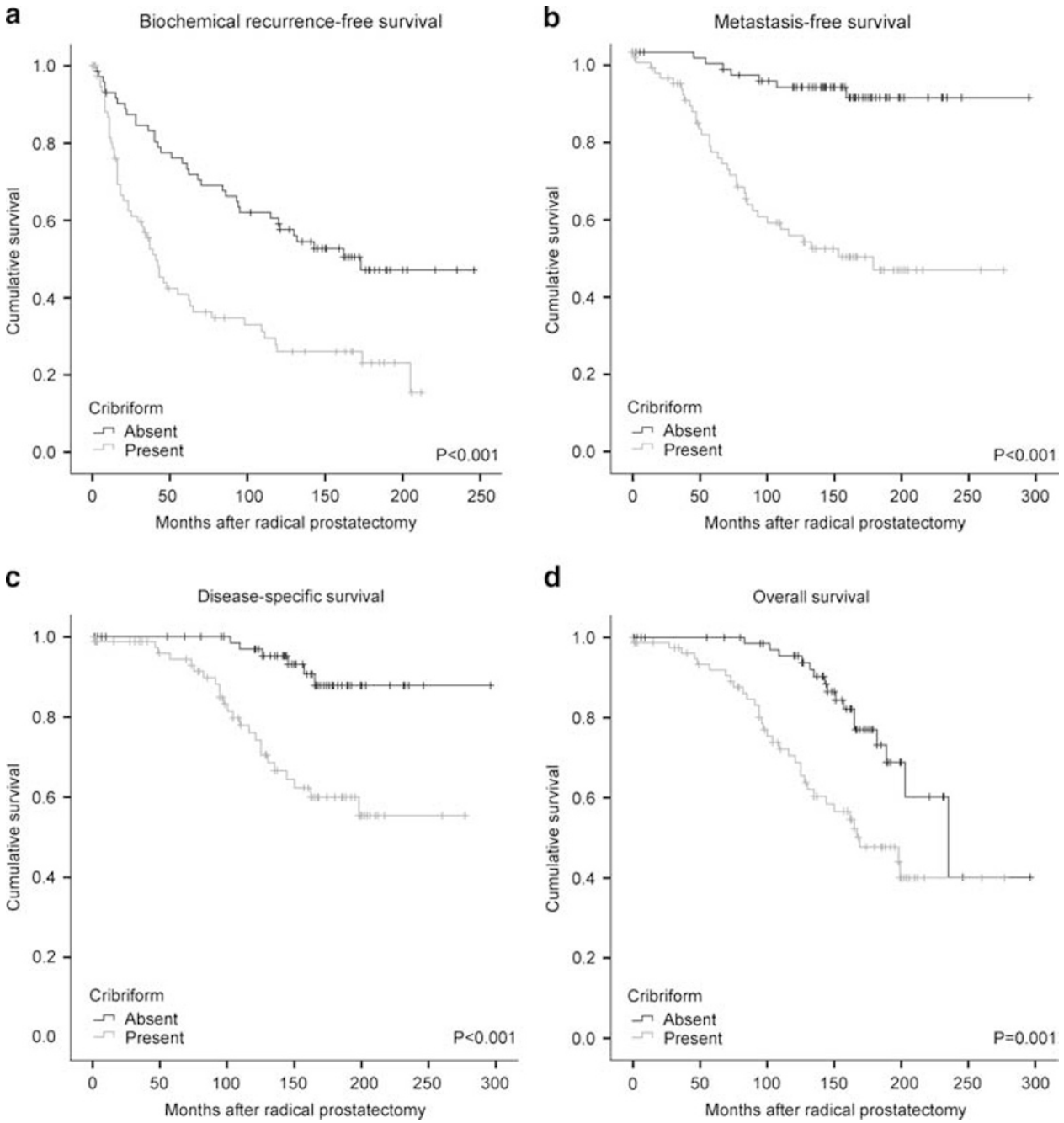

Figure 2 Kaplan-Meier estimates on impact of cribriform growth pattern in (a) biochemical recurrence-free survival; (b) distant metastasis-free survival; (c) disease-specific survival; and (d) overall survival.

encompasses a heterogeneous group of growth patterns, defined as fused, ill-defined, cribriform, and glomeruloid. ${ }^{2,5}$ Our objective was to determine the prognostic value of individual Gleason grade 4 patterns in Gleason score 7 prostate cancer patients.

In this study, we found that the presence of cribriform pattern in radical prostatectomy specimens was a major predictive factor for distant metastasis and disease-specific death of prostate cancer. In fact, cribriform pattern was the strongest predictor for both adverse clinical events in multivariate analysis, adjusted not only for established clinicopathologic parameters (age, PSA, Gleason score, pT stage, and surgical margins), but also for contemporary additional pathologic parameters such as intraductal carcinoma and tertiary Gleason grade 5 pattern. Therefore, identification of cribriform growth in daily pathology practice is a new, fast, and cheap adjunct to predict adverse clinical outcome.

The clinical significance of Gleason grade 4 patterns has only since recently become an area of interest. Dong et $a^{19}$ found that metastasis occurred five times more frequently in patients with cribriform pattern at radical prostatectomy $(13 \% \mathrm{vs}$ $2.6 \%$ when cribriform pattern is absent) in a consecutive series of 241 patients. These results are well in line with the findings of the current study. However, Dong et a $1^{19}$ did not take into account important parameters, such as lymph node status, intraductal carcinoma, and tertiary Gleason grade 5.

A strong point of this study is the use of metastasis-free and disease-specific survival as end points as they objectively reflect aggressive tumor biology, whereas biochemical and local recurrence also depend on surgical margin status and do not necessarily indicate metastatic tumor potential. In addition, we included contemporary pathologic characteristics such as intraductal carcinoma and tertiary Gleason grade 5 as covariates in our analysis. It is striking that the presence of cribriform growth either as invasive prostate cancer or as intraductal expansion is associated with disease out- 
Table 3 Hazard ratios for metastasis after radical prostatectomy

\begin{tabular}{|c|c|c|c|c|c|c|c|c|}
\hline \multirow{2}{*}{ Parameter } & \multicolumn{4}{|c|}{ Distant metastasis } & \multicolumn{4}{|c|}{ Disease-specific death } \\
\hline & $\begin{array}{c}\text { Crude HR } \\
\text { (95\% CI) }\end{array}$ & P-value & $\begin{array}{l}\text { Adjusted HR } \\
(95 \% \text { CI })\end{array}$ & P-value & $\begin{array}{c}\text { Crude HR } \\
(95 \% \text { CI) }\end{array}$ & P-value & $\begin{array}{l}\text { Adjusted HR } \\
(95 \% \mathrm{CI})\end{array}$ & $\mathrm{P}$-value \\
\hline Age & $1.0(0.97-1.1)$ & 0.39 & $1.0(0.97-1.1)$ & 0.33 & $1.0(0.95-1.1)$ & 0.67 & $1.0(0.92-1.1)$ & 0.91 \\
\hline PSA level & $1.0(0.98-1.0)$ & 0.44 & $1.0(0.98-1.0)$ & 0.63 & $1.0(0.99-1.1)$ & 0.18 & $1.0(1.0-1.1)$ & 0.058 \\
\hline Gleason score $4+3=7$ & $2.5(1.3-4.9)$ & 0.005 & $1.2(0.52-2.7)$ & 0.60 & $4.1(2.0-8.4)$ & $<0.001$ & $3.1(1.4-7.1)$ & 0.007 \\
\hline Seminal vesicle invasion & $2.3(0.87-6.2)$ & 0.092 & $1.3(0.44-4.1)$ & 0.59 & $0.71(0.23-2.2)$ & 0.56 & $0.12(0.19-1.2)$ & 0.12 \\
\hline Positive surgical margin & $1.1(0.56-2.2)$ & 0.79 & $0.76(0.37-1.6)$ & 0.46 & $0.84(0.39-1.8)$ & 0.65 & $0.57(0.21-1.6)$ & 0.27 \\
\hline Positive lymph node status & $2.3(0.71-7.7)$ & 0.16 & $1.4(0.39-5.2)$ & 0.60 & $0.94(0.13-6.9)$ & 0.94 & $0.50(0.057-4.4)$ & 0.54 \\
\hline Fused & $0.86(0.43-1.7)$ & 0.68 & $0.47(0.22-1.0)$ & 0.048 & $0.93(0.43-2.0)$ & 0.85 & $0.55(0.23-1.3)$ & 0.18 \\
\hline Ill-defined & $0.62(0.32-1.2)$ & 0.14 & $0.82(0.38-1.7)$ & 0.74 & $0.82(0.40-1.7)$ & 0.60 & $2.0(0.77-5.3)$ & 0.15 \\
\hline Cribriform & $6.8(2.8-16)$ & $<0.001$ & $8.0(3.0-21)$ & $<0.001$ & $5.0(2.1-12)$ & $<0.001$ & $5.4(2.0-15)$ & 0.001 \\
\hline Glomeruloid & $0.52(0.20-1.3)$ & 0.17 & $0.53(0.18-1.6)$ & 0.25 & $0.73(0.28-1.9)$ & 0.52 & $0.80(0.24-2.7)$ & 0.82 \\
\hline Intraductal carcinoma & $2.5(1.2-4.7)$ & 0.007 & $0.91(0.39-2.1)$ & 0.89 & $2.3(1.1-4.7)$ & 0.028 & $1.0(0.40-2.7)$ & 0.93 \\
\hline Tertiary Gleason grade 5 & $2.8(1.1-7.2)$ & 0.034 & $1.6(0.44-5.4)$ & 0.49 & $1.4(0.32-5.7)$ & 0.67 & $\mathrm{n} / \mathrm{a}$ & \\
\hline
\end{tabular}

Values are adjusted for age; PSA level; Gleason score; pT stage; positive surgical margin; positive lymph node at time of surgery; Gleason grade 4 patterns; intraductal carcinoma and tertiary Gleason grade 5.

come. In both cribriform pattern and intraductal carcinoma, a majority of tumor cells has lost physical contact with surrounding stromal matrix and basement membrane, respectively, and only connect to adjacent epithelial tumor cells. The presence of intraductal carcinoma has been associated with high-grade cancer and adverse outcome. ${ }^{13-15}$ In our study, presence of cribriform pattern was significantly associated with intraductal carcinoma. Two hypotheses of intraductal carcinoma evolution have been proposed. ${ }^{20}$ First, established Gleason grade 4 or 5 prostate cancer could infiltrate and expand preexisting glands; this theory is supported by the fact that intraductal carcinoma is rarely found in absence of high-grade prostate cancer. ${ }^{21}$ Alternatively, high-grade prostatic intraepithelial neoplasia, which is generally accepted as prostate cancer precursor, might evolve into intraductal carcinoma and consecutively invasive high-grade prostate cancer. The molecular and biological connections of separate intraductal and invasive growth patterns are intriguing and clinically important, but still poorly understood.

A limitation of the current study is the nested case-control design, in which the cases had Gleason score $4+3=7$ prostate cancer more often than the controls. Although Cox regression analysis compensated for the unequal Gleason score distribution, we performed additional analysis in $3+4$ and $4+3$ prostate cancer subgroups, which confirmed the prognostic value of cribriform pattern. Another caveat of our study was the strict delimitation of Gleason grade 4 patterns in four groups. This subdivision reflects the categories defined by the ISUP/WHO, but does not take into account subtle architectural variations such as small and large cribriform fields, locally fused glands, and complex fused structures with intervening stroma. In this study, we did not determine the relative percentage or volume of Gleason grade 4. Although subgroup analysis of cribriform growth in Gleason score $3+4=7 \quad(<50 \%$ Gleason grade 4$)$ and $4+3=7$ ( $\geq 50 \%$ Gleason grade 4) revealed independent prognostic value in both groups, further delineating of Gleason grade 4 percentage might have influenced outcome. Interestingly, Ross et $a l^{8}$ suggested in their large study of lymph node metastasis that even the slightest presence of cribriform growth could give rise to metastasis. Furthermore, we did not take prostate cancer volume or an associated parameter into account. Although prostate cancer volume might be a confounder in our study, our group has previously shown that it did not add prognostic value to established pathologic parameters. ${ }^{22}$ We matched for $\mathrm{pT}$ stage as a surrogate of prostate cancer volume in this study.

The outcome of our study is of significant clinical relevance. Currently, patients with Gleason score $3+4=7$ are considered to be at low to intermediate risk in clinical practice; they even might be candidates for active surveillance. However, based on our results, Gleason score 7 patients with cribriform growth are more likely to be candidates for treatment. If validated on diagnostic prostate needle-biopsies, cribriform growth might influence therapeutic decision making in clinical practice.

\section{Conclusion}

Cribriform growth is a novel and strong independent histopathological predictor for distant metastasisfree survival and disease-specific survival in patients with Gleason score 7 prostate cancer at radical prostatectomy.

\section{Disclosure/conflict of interest}

The authors declare no conflict of interest. 


\section{References}

1 Gleason DF. Classification of prostatic carcinomas. Cancer Chemother Rep 1966;50:125-128.

2 Epstein JI, Allsbrook WC Jr, Amin MB, et al. Committee IG. The 2005 international society of urological pathology (ISUP) consensus conference on Gleason grading of prostatic carcinoma. Am J Surg Pathol 2005;29:1228-1242.

3 Lotan TL, Epstein JI. Gleason grading of prostatic adenocarcinoma with glomeruloid features on needle biopsy. Hum Pathol 2009;40:471-477.

4 Latour M, Amin MB, Billis A, et al. Grading of invasive cribriform carcinoma on prostate needle biopsy: An interobserver study among experts in genitourinary pathology. Am J Surg Pathol 2008;32:1532-1539.

5 Epstein JI. Update on the Gleason grading system. Ann Pathol 2011;31:S20-S26.

6 Falzarano SM, Magi-Galluzzi C. Prostate cancer staging and grading at radical prostatectomy over time. Adv Anat Pathol 2011;18:159-164.

7 Hernandez DJ, Nielsen ME, Han M, et al. Natural history of pathologically organ-confined (pT2), Gleason score 6 or less, prostate cancer after radical prostatectomy. Urology 2008;72:172-176.

8 Ross HM, Kryvenko ON, Cowan JE, et al. Do adenocarcinomas of the prostate with Gleason score (GS) $</=6$ have the potential to metastasize to lymph nodes? Am J Surg Pathol 2012;36:1346-1352.

9 Eggener SE, Scardino PT, Walsh PC, et al. Predicting 15-year prostate cancer specific mortality after radical prostatectomy. J Urol 2011;185:869-875.

10 Sobin LH, Gospodarowicz MK, Wittekind C. TNM classification of malignant tumours, 7th edn. Wiley: Oxford, 2009, 336p.

11 Gleason DF, Mellinger GT. Prediction of prognosis for prostatic adenocarcinoma by combined histological grading and clinical staging. J Urol 1974;111:58-64.

12 Gleason DF. Histologic grading of prostate cancer: A perspective. Hum Pathol 1992;23:273-279.
13 Cohen RJ, McNeal JE, Baillie T. Patterns of differentiation and proliferation in intraductal carcinoma of the prostate: Significance for cancer progression. Prostate 2000;43:11-19.

14 Van der Kwast T, Al Daoud N, Collette L, et al. Biopsy diagnosis of intraductal carcinoma is prognostic in intermediate and high risk prostate cancer patients treated by radiotherapy. Eur J Cancer 2012;48: 1318-1325.

15 Efstathiou E, Abrahams NA, Tibbs RF, et al. Morphologic characterization of preoperatively treated prostate cancer: Toward a post-therapy histologic classification. Eur Urol 2010;57:1030-1038.

16 Hattab EM, Koch MO, Eble JN, et al. Tertiary Gleason pattern 5 is a powerful predictor of biochemical relapse in patients with Gleason score 7 prostatic adenocarcinoma. J Urol 2006;175:1695-1699.

17 Whittemore DE, Hick EJ, Carter MR, et al. Significance of tertiary Gleason pattern 5 in Gleason score 7 radical prostatectomy specimens. J Urol 2008;179:516-522.

18 Pickup M, Van der Kwast TH. My approach to intraductal lesions of the prostate gland. J Clin Pathol 2007;60:856-865.

19 Dong F, Yang P, Wang C, et al. Architectural heterogeneity and cribriform pattern predict adverse clinical outcome for Gleason grade 4 prostatic adenocarcinoma. Am J Surg Pathol 2013;12:1855-1861.

20 Cohen RJ, Wheeler TM, Bonkhoff $\mathrm{H}$, et al. A proposal on the identification, histologic reporting, and implications of intraductal prostatic carcinoma. Arch Pathol Lab Med 2007;131:1103-1109.

21 McNeal JE, Yemoto CE. Spread of adenocarcinoma within prostatic ducts and acini. morphologic and clinical correlations. Am J Surg Pathol 1996;20: 802-814.

22 Wolters T, Roobol MJ, van Leeuwen PJ, et al. Should pathologists routinely report prostate tumour volume? The prognostic value of tumour volume in prostate cancer. Eur Urol 2010;57:821-829. 